\title{
Comparative study of gene expression by cDNA microarray in human colorectal cancer tissues and normal mucosa
}

\author{
MICHELE BIANCHINI $^{1}$, ESTRELLA LEVY ${ }^{1,2}$, CINZIA ZUCCHINI $^{3}$, VICTOR PINSKI ${ }^{2,5}$, CARLOS MACAGNO $^{2}$, \\ PAOLA DE SANCTIS ${ }^{3}$, LUISA VALVASSORI ${ }^{3}$, PAOLO CARINCI ${ }^{3}$ and JOSÉ MORDOH ${ }^{4}$ \\ ${ }^{1}$ Centro de Investigaciones Oncológicas (CIO-FUCA); ${ }^{2}$ Maestría en Biología Molecular Médica, \\ Universidad de Buenos Aires, Argentina; ${ }^{3}$ Dipartimento di Istologia, Embriologia e Biologia Applicata, \\ Università degli Studi di Bologna, Via Belmeloro 8, I-40126 Bologna, Italy; ${ }^{4}$ Laboratorio de Cancerología \\ Fundacion Instituto Leloir; ${ }^{5}$ M.B. de Martinez Hospital, Buenos Aires, Argentina
}

Received November 14, 2005; Accepted January 30, 2006

\begin{abstract}
The causative molecular pathways underlying the pathogenesis of colorectal cancer (CRC) need to be better characterized. The purpose of our study was to better understand the genetic mechanism of oncogenesis for human colorectal cancer and to identify new potential tumor markers of use in clinical practice. We used cDNA microarrays to compare gene expression profiles of colorectal biopsies from 25 CRC patients and 13 normal mucosa from adjacent non-cancerous tissues. Findings were validated by real-time PCR; in addition, Western blotting and immunochemistry analysis were carried out as further confirmation of differential expression at a protein level. Comparing cancerous tissues with normal colonic mucosa we identified 584 known genes differentially expressed to a significant degree $(p<0.001)$. Many of the transcripts that were more abundant in tumors than in non-neoplastic tissues appear to reflect important events for colon carcinogenesis. For example, a significant number of these genes serve as apoptotic inhibitors (e.g. BFAR, BIRC1, BIRC6). Furthermore, we observed the simultaneous up-regulation of HLA-E and the down-regulation of $\beta 2$-microglobulin; these genes strongly support a potential tumor escape strategy from immune surveillance in colon cancer tissues. Our study provides new gene candidates in the pathogenesis of human CRC disease. From our results we hypothesize that CRC cells escape immune surveillance through a specific gene expression alteration; moreover, over-expression of several survival genes seems to confer a more anti-apoptotic phenotype. These genes are involved in pathways not previously implicated in CRC pathogenesis and they may provide new targets for therapy.
\end{abstract}

Correspondence to: Professor Paolo Carinci, Dipartimento di Istologia, Embriologia e Biologia Applicata, Università degli Studi di Bologna, Via Belmeloro 8, I-40126 Bologna, Italy

E-mail: carinci@alma.unibo.it

Key words: colon cancer, cDNA microarray, gene expression profiles, anti-apoptotic phenotype

\section{Introduction}

Colorectal cancer (CRC) is the third most common cancer in both men and women (1). Despite several important advances in treatment and diagnosis, which have resulted in a doubling in the survival rate over the last 20 years, this disease remains one of the most frequent and deadly neoplasias in the Western world $(2,3)$. The incidence and mortality of colorectal cancer increase with age, especially after 60 years of age (4). Colorectal cancer is a heterogeneous disease arising from a complex series of molecular changes. The evolution of normal colonic mucosa to a benign adenoma, then to dysplastic cells, and finally to a potentially invasive cancer is associated with a series of genetic events occurring over a long period (5). Furthermore, as with many other cancers, the development of colorectal cancer typically results from a complex interaction between genetic and environmental influences (diet, medications and life style) (6). Molecular detection methods based on gene mutation determination for APC, p53 and K-ras, have been carried out for over a decade (7). Despite the usefulness of these molecular markers, the applications remain limited for CRC patients; for this reason, new molecular markers are needed to improve diagnosis, prognosis and treatment. Microarray technology simultaneously providing information on expression levels of thousands of genes has revealed new potential diagnostic biomarkers and molecular targets for known chemotherapeutic agents and also for novel tumor treatment strategies $(8,9)$. Recent studies suggest that DNA microarray profiling performed on clinical specimens may provide information directly applicable to cancer diagnosis $(10,11)$. In addition to cancer diagnosis, gene profiling is being explored as a means of predicting tumor treatment response (12).

In our study, we compared gene expression profiles of whole colorectal cancer tissues and adjacent non-cancerous mucosa from surgical resections, in order to improve our understanding of the genetic mechanism of oncogenesis for human colorectal cancer and to identify new potential tumor markers useful for clinical practice. In particular, use of cDNA microarray to assess the expression of approximately 19,200 genes in 25 sporadic colorectal carcinomas (Dukes' B and C) 
gave us new insights into the genetic mechanisms underlying neoplastic transformation for colorectal cells.

\section{Materials and methods}

Patients and tissue samples. A total of 82 pairs of colorectal cancer tissues and corresponding adjacent non-cancerous tissues were obtained from 41 patients with sporadic colorectal adenocarcinoma who underwent surgical resection at the J. Mendez Hospital and M.B. de Martinez Hospital (Buenos Aires, Argentina) between May 2003 and June 2004. The Institutional Review Boards of both institutions approved this research. Each resected cancer specimen was evaluated for its tumor cell content in hematoxylin and eosin stained sections. Only specimens containing $>75 \%$ tumor cells were used for analysis. Samples were macrodissected by pathologists and within $30 \mathrm{~min}$ of removal frozen in liquid nitrogen for molecular analysis. Informed consent was obtained from each patient.

RNA extraction and cDNA preparation. Total RNA was extracted from frozen samples using TRIzol reagent (Invitrogen Life Technology Inc., USA) as recommended by the manufacturer. DNA was digested with RNase-free DNase I for 30 min at $37^{\circ} \mathrm{C}$ using a DNA-free ${ }^{\mathrm{TM}}$ kit (Ambion Inc., USA); following digestion, DNase was then inactivated and removed using DNase inactivation reagent included in the kit. The quality of RNA samples was determined by agarose gel electrophoresis. Only 38 samples, from our tumor bank, which yielded high quality RNA with minimal degradation and clear $28 \mathrm{~S} / 18 \mathrm{~S}$ ribosomal bands, were included in the analysis. Twenty-five tumors of different clinical stage, in addition to 13 normal mucosa specimens, were used for microarray experiments. Normal samples were pooled in equimolar amounts to generate a single standard reference RNA employed as a control in each microarray hybridization. LabelStar Array Kit (Qiagen, CA, USA) was used to obtain labeled cDNA. For half of microarray hybridizations, $50 \mu \mathrm{g}$ of total RNA isolated from each tumor sample were retrotranscribed and labeled with Cy5-dCTP (Perkin-Elmer Life Science, USA) and cDNA obtained from $50 \mu \mathrm{g}$ of the standard reference RNA was labeled with Cy3-dCTP (Perkin-Elmer Life Science); the fluorochromes were inverted in the second half of microarrays with the purpose of reducing dye bias.

Microarray hybridization and image acquisition. Hybridization experiments were performed on SS-Human 19Kv7 microarray (University Health Network Microarray Centre, Ontario, Canada) containing 19,200 probes derived from coding and EST sequences deposited in GenBank. Equivalent amounts of Cy5-cDNA and Cy3-cDNA were combined and hybridized to microarray slides for approximately $18 \mathrm{~h}$ at $37^{\circ} \mathrm{C}$. The slides were washed three times with $1 \mathrm{X} \mathrm{SSC}+0.1 \%$ SDS (pre-warmed to $50^{\circ} \mathrm{C}$ ) for $30 \mathrm{~min}$ then twice with $1 \mathrm{X} \mathrm{SSC}$ for $10 \mathrm{~min}$ and finally once for $10 \mathrm{~min}$ with $0.1 \mathrm{X}$ SSC. Hybridized slides were scanned using the GenePix 4000a Scanner (Axon Instruments, Foster City, CA; facility at the Dipartimento di Istologia, Embriologia e Biologia Applicata, Bologna University, Bologna, Italy) and images acquired by GenePix Pro v5.0 software.
Data analysis. Twenty-five microarray data sets were sorted and loaded on ExpressConverter v1.5, which enabled us to transform GenePix generated result files into the correct file format (.mev) to be properly used with MIDAS v2.18 (Microarray Data Analysis System) software from TIGR (The Institute for Genomic Research). MIDAS was used to preprocess and normalize (within-slide LOWESS normalization) the raw microarray data; moreover, it creates output for $\mathrm{MeV}$ v3.0 (MultiExperiment Viewer) software from TIGR, an application that allows statistical analysis and the identification of genes and expression patterns of interest. Data mining was carried out by EASE (Expression Analysis Systematic Explorer; SAIC-Frederick Inc. Clinical Services Program, Laboratory of Immunopathogenesis and Bioinformatics) which is a powerful tool for rapidly converting the results of functional genomic studies from 'genes to themes' and to automate the process of biological theme determination.

Statistical methods. Using the $\log _{2}$ ratios of selected genes the reference list was generated by using Student's t-test statistics calculated on the full set of 21 patients. Significantly different expression between tumor and normal mucosa was estimated using a one-class t-test; gene's p-value was determined by forming a distribution based on 10,000 random permutations of the data. The p-value for each gene was scaled by Adjusted Bonferroni Correction, with the aim of correcting for the large number of observations and the consequently increased possibility of considering a gene without a real significant change to be considered significant. We defined those genes with adjusted $\mathrm{p}<0.001$ as informative.

EASE was run using ease score (es) as default metric to rank categories of genes by over-representation. This score is the upper bound of the distribution of Jackknife Fisher exact probabilities. This statistical measure (adjusted by the Bonferroni method) of over-represented gene clusters favors more robust categories than the Fisher exact probability.

Real-time PCR. To confirm the cDNA microarray results, real-time PCR was performed on 6 patients for 10 genes displaying a clear change in their expression. Reverse transcription was performed on $5 \mu \mathrm{g}$ of total RNA for 50 min at $42^{\circ} \mathrm{C}$ using an oligo(dT)24 primer and Superscript II reverse transcriptase (Invitrogen Life Technology Inc.). Real-time PCR analysis was performed on selected genes using the set of primers reported in Table I. Triple determinations were performed on an ICycler $^{\circledR}$ from Bio-Rad using Platinum Taq DNA Polymerase and SYBR Green (Invitrogen Life Technology Inc.); GAPDH and actin- $\beta$ levels were determined to normalize all samples. Amplification efficiency and error were estimated for each pair of primers by standard curve calculation (derived from serial dilutions of positive cDNA).

Western blot analysis. To perform Western blot analysis we used the monoclonal antibody MEM-E/02 (BioVendor Laboratory Medicine, Inc., Czech Republic) to HLA-E molecule. Homogenization of tissue samples was carried out in $0.5 \mathrm{ml}$ of lysis buffer (50 mM Tris-HCl, pH $8.0150 \mathrm{mM}$ $\mathrm{NaCl}, 0.1 \%$ SDS, $0.5 \%$ DCS, $1 \%$ NP-40, 5 mM EDTA) containing a protease inhibitor cocktail for mammalian tissues (Sigma, USA). Protein lysates were supplemented with 4X 
Table I. Primers of GAPDH, actin- $\beta$ (housekeeping genes) and 10 deregulated genes confirmed with quantitative reverse transcription-PCR.

\begin{tabular}{|c|c|c|}
\hline Gene name & Accession number & Forward and reverse primers \\
\hline MT1G & NM_005950 & $\begin{array}{l}\text { 5'-TCT CGC TTG GGA ACT CTA GTC-3' } \\
\text { 5'-TTG CAG GAG GTG CAT TTG-3' }\end{array}$ \\
\hline B2M & NM_004048 & $\begin{array}{l}\text { 5'-GCT CGC GCT ACT CTC TCT TT-3' } \\
\text { 5'-ATT CTC TGC TGG ATG ACG TG-3' }\end{array}$ \\
\hline SMURF2 & NM_022739 & $\begin{array}{l}\text { 5'-CTC GGC TGT CTG CTA ACT TG-3' } \\
\text { 5'-CGA TAC CAC TTG CTG TTG CT-3' }\end{array}$ \\
\hline EIF4EL3 & NM_004846 & $\begin{array}{l}\text { 5'-GTG GTG TCT GTC CGC TTT C-3' } \\
\text { 5'-ATG GTG TTG GGA GGT AGG TT- 3' }\end{array}$ \\
\hline CASP3 & NM_004346 & $\begin{array}{l}\text { 5'-TAG ATG GTT TGA GCC TGA GC-3' } \\
\text { 5'-CAG TGC GTA TGG AGA AAT GG-3' }\end{array}$ \\
\hline TIMP3 & NM_000362 & $\begin{array}{l}\text { 5'-TTGCCCTTCTCCTCCAATAC-3' } \\
\text { 5'-CTATCTGCTTGCTGCCTTTG-3' }\end{array}$ \\
\hline DSC3 & NM_001941 & $\begin{array}{l}\text { 5'-CTTCTTCTGAGTGGCATTGG-3' } \\
\text { 5'-GGAATCTCTGCTGGAGGTGT-3' }\end{array}$ \\
\hline PIK3R2 & NM_005027 & $\begin{array}{l}\text { 5'-TTGGAAGAGCAGGAGGTTG-3' } \\
\text { 5'-TGGCGGTAGTGATTGATGAG-3' }\end{array}$ \\
\hline SLC16A1 & NM_003051 & $\begin{array}{l}\text { 5'-ATGCTGTCCTGTCCTCCTG-3' } \\
\text { 5'-GCCCTCCTTCTGTGTCTTTC-3' }\end{array}$ \\
\hline F11R & NM_144501 & $\begin{array}{l}\text { 5'-CCTATGTCCTGAATCCCACA-3' } \\
\text { 5'-GGCTGGCTGTAAATCACCTT-3' }\end{array}$ \\
\hline ACTB & NM_001101 & $\begin{array}{l}\text { 5'-CACTCTTCCAGCCTTCCTTC-3' } \\
\text { 5'-TACAGGTCTTTGCGGATGTC-3' }\end{array}$ \\
\hline GAPDH & NM_002046 & $\begin{array}{l}\text { 5'-TCAAGAAGGTGGTGAAGCAG-3' } \\
\text { 5'-CGTCAAAGGTGGAGGAGTG-3' }\end{array}$ \\
\hline
\end{tabular}

sample buffer $(250 \mathrm{mM}$ Tris- $\mathrm{HCl} \mathrm{pH} 6.8,0.8 \%$ SDS, $40 \%$ glycerol, $0.7 \mathrm{M}$ 2- $\beta$ mercaptoetanol) and heated for $5 \mathrm{~min}$ at $95^{\circ} \mathrm{C}$; equal amounts of total protein lysate from either tumor tissues and normal colonic mucosa were loaded in each lane and separated in $15 \%$ SDS-PAGE; proteins were then electroblotted onto nitrocellulose membranes of $0.45 \mu \mathrm{m}$ pore size and the membranes blocked by incubation with PBS containing $0.2 \%$ Tween-20 and 5\% BSA. Following homogenization, protein lysates were quantified by Bradford method, separated by electrophoresis and finally transferred onto nitrocellulose membrane. The membranes were probed with anti HLA-E antibody (diluted 1:3,000) and anti-GAPDH (Ambion Inc.) as loading control protein diluted 1:10,000 for $1 \mathrm{~h}$ at room temperature. After washing, membranes were incubated for $45 \mathrm{~min}$ at room temperature with goat anti-mouse alkaline phosphatase (Jackson Immunoresearch Lab. Inc., USA) and washed thoroughly. Signals were detected using BCIP/NBT color development substrate (Promega, USA).

Immunochemistry analysis. Monoclonal antibody to HLA-E (BioVendor Laboratory Medicine) was applied on colon tissue fixed sections using an indirect immunostaining technique. In brief, section were deparaffinized and hydrated, endogenous peroxidase activity was quenched by incubation with $2 \% \mathrm{H}_{2} \mathrm{O}_{2}$ in methanol for $10 \mathrm{~min}$. Rehydrated sections were incubated overnight at $4^{\circ} \mathrm{C}$ with primary antibody and for $30 \mathrm{~min}$ at room temperature with biotinylated anti-mouse $\operatorname{IgG}$ diluted in PBS containing $1 \%$ normal horse serum. The immunostaining reaction was developed using Vectastain ${ }^{\circledR} \mathrm{ABC}$ Reagent (Vector Laboratories, Inc., USA) and NovaRED ${ }^{\mathrm{TM}}$ substrate solution (Vector Laboratories, Inc.). Sections were finally counterstained with hematoxylin, dehydrated and permanently mounted in non-aqueous mounting media.

\section{Results}

The mRNA expression profiles of 25 primary human colorectal tumor tissues were determined by comparison with a reference RNA pool from normal mucosa using 19,200element cDNA microarrays; Table II illustrates the clinical characteristics of patients in this study.

In our experimental design, cDNA arrays were used as a two-label system in which two RNA samples were separately labeled, mixed, and hybridized together on each array. 
Table II. Clinical and histological characteristics of analysed patients.

\begin{tabular}{|c|c|c|c|c|c|c|c|c|}
\hline Patient no. & Sex & Age & Location & Grade $^{a}$ & TNM & Dukes & Lymph node & AJCC stage \\
\hline 1 & M & 65 & Right & M & T3N1M0 & $\mathrm{C}$ & Yes & 3 \\
\hline 4 & M & 49 & Rectum & $\mathrm{G}$ & T3N2M0 & $\mathrm{C}$ & Yes & 3 \\
\hline 5 & $\mathrm{M}$ & 50 & Rectum & M & T3N1M0 & $\mathrm{C}$ & Yes & 3 \\
\hline 7 & M & 71 & Rectum & $\mathrm{P}$ & T3N2M0 & $\mathrm{C}$ & Yes & 3 \\
\hline 12 & M & 72 & Right & G & T3N2M0 & $\mathrm{C}$ & Yes & 3 \\
\hline 15 & M & 77 & Left & G & T1N0M0 & B & No & 1 \\
\hline 16 & $\mathrm{~F}$ & 83 & Rectum & M & T2NOM0 & B & No & 1 \\
\hline 17 & $\mathrm{~F}$ & 81 & Right & $\mathrm{M}$ & T3N1M0 & $\mathrm{C}$ & No & 3 \\
\hline 21 & M & 77 & Right & M & T3N1M0 & $\mathrm{C}$ & Yes & 3 \\
\hline 22 & $\mathrm{~F}$ & 78 & Right & $\mathrm{P}$ & T3N0M0 & $\mathrm{C}$ & No & 2 \\
\hline 24 & $\mathrm{~F}$ & 35 & Right & $\mathrm{M}$ & T3N1M0 & $\mathrm{C}$ & Yes & 3 \\
\hline 26 & M & 82 & Left & $\mathrm{G}$ & T3NOMO & $\mathrm{B}$ & No & 2 \\
\hline 28 & M & 78 & Left & $\mathrm{P}$ & T3N2M0 & $\mathrm{C}$ & Yes & 3 \\
\hline 29 & $\mathrm{~F}$ & 61 & Left & $\mathrm{M}$ & T3NOM0 & $\mathrm{C}$ & No & 2 \\
\hline 30 & M & 86 & Right & $\mathrm{G}$ & T3N1M0 & $\mathrm{C}$ & Yes & 3 \\
\hline 31 & $\mathrm{~F}$ & 81 & Right & $\mathrm{G}$ & T3N1M0 & $\mathrm{C}$ & Yes & 3 \\
\hline 32 & M & 69 & Left & $\mathrm{G}$ & T3N0M0 & B & No & 2 \\
\hline 33 & $\mathrm{~F}$ & 89 & Right & M & T3N1M0 & $\mathrm{C}$ & Yes & 3 \\
\hline 34 & $\mathrm{~F}$ & 77 & Right & $\mathrm{G}$ & T3N0M0 & B & Yes & 2 \\
\hline 35 & $\mathrm{~F}$ & 78 & Right & $\mathrm{G}$ & T3N0M0 & B & No & 2 \\
\hline 36 & M & 75 & Rectum & M & T1NOM0 & B & Yes & 1 \\
\hline 37 & $\mathrm{M}$ & 56 & Rectum & $\mathrm{M}$ & T1N0M0 & B & No & 1 \\
\hline 39 & $\mathrm{M}$ & 73 & Rectum & $\mathrm{G}$ & T2N0M0 & B & No & 1 \\
\hline 40 & M & 76 & Left & $\mathrm{G}$ & T3N1M0 & $\mathrm{C}$ & Yes & 3 \\
\hline 41 & $\mathrm{M}$ & 68 & Rectum & $\mathrm{M}$ & T3N2M0 & $\mathrm{C}$ & Yes & 3 \\
\hline
\end{tabular}

${ }^{\mathrm{a}}$ Grade: G, good; M, moderate; P, poor. ${ }^{\mathrm{b}}$ Lymph node: yes, regional lymph node invasion; no, absent regional lymph node invasion.

Fluorescence intensity data were acquired and stored for 25 microarrays representing 25 colorectal tumor specimens compared to normal mucosa. Acquired data were initially pre-processed by image analysis using GenePix software; then with the aim of selecting solid reliable data, arrays were excluded from the analysis when $>50 \%$ of all genes were accompanied by 'absent' or 'bad' flags. The remaining 21 microarrays were further processed using MIDAS software. With the purpose to enable other users to comprehensively interpret and evaluate our results, original tables of complete microarray results are available in the supplementary data (see the GEO website at http://www.ncbi.nlm.nih.gov/ projects/geo/; accession number: GSE3294). The application of MIDAS criteria led us to select 4,095 sequences differentially expressed in tumor samples as compared to normal mucosa $(21 \%$ of total genes and ESTs present in each initial array). Approximately $50 \%$ of these are known genes.

Functional categorization. We used EASE to assign the differentially expressed sequences to functional categories. A cut-off filtering criterion was employed: we considered only those genes with an average change (mean of 21 values) $>1.5$-fold induction or repression (1,306 genes). As expected, genes chosen for functional categorization reflect the heterogeneity in cell composition of colon samples. In addition to genes belonging to colon epithelial cells, we found genes that are known to be expressed in stromal cells (e.g. COL1A2, MMP2, TIMP3) smooth muscle cells (e.g. CNN3, MYH9) and immune system cells (e.g. DEFB1, IGHM). A high percentage of over-expressed genes in cancer tissue related to inositol/phosphatidylinositol kinase activity (es $=0.006$ ) and apoptosis inhibitor activity (es $=0.022$ ) (Table III). On the other hand, decreased genes in cancer tissue belonged predominantly to the mitochondrion $(\mathrm{es}=0.005)$ and ribosome clusters (es $=0.008)$ (Table III).

Global expression analysis of genes in CRC patients. In an effort to reduce the large dataset obtained by application of MIDAS criteria (4,095 cDNA clones) we carried out a statistical analysis based on a one-class t-test design. We were thus able to identify 584 known genes (3\% of total genes and ESTs present in each initial array) differentially expressed to a significant degree $(\mathrm{p}<0.001)$ in colon cancer compared to non-neoplastic colon mucosa. Here, we report a list (Table IV) limited to those genes showing at least an average fold change $\geq 2$ ( 88 genes, 76 of which up-regulated and 12 down-regulated). Many of the transcripts that were more abundant in tumors than in non-neoplastic tissues, appear to reflect important events for colon carcinogenesis (Fig. 1): for example ITGA2 and VEGF which are known 
Table III. Functional categories of known genes with $>1.5$-fold increasing (up) or decreasing (down) expression from tumor to normal tissue.

\begin{tabular}{|c|c|c|}
\hline Functional category & EASE score ${ }^{a}$ & Gene population $^{\mathrm{b}}$ \\
\hline \multicolumn{3}{|l|}{ Up-regulated categories } \\
\hline Inositol/phosphatidylinositol kinase activity & 0.006 & ATM; FRDA; PIK3R1; PIK3R2; PIP5K1A \\
\hline Apoptosis inhibitor activity & 0.022 & API5; BAG1; BFAR; BIRC1; BIRC6 \\
\hline Lipid transporter activity & 0.047 & APOC1; ATP8A2; HDLBP; PITPNB; STARD4 \\
\hline \multicolumn{3}{|l|}{ Down-regulated categories } \\
\hline Mitochondrion & 0.005 & $\begin{array}{l}\text { ATP5C1; ATP5G2; ATP5J2; ATP5S; BCKDHA; } \\
\text { BRPF3; CLPX; COX4I1; COX6C; COX7A2; COX7B; } \\
\text { CPT1B; CYB5-M; CYBB; DAP3; DECR1;DLAT; } \\
\text { ECH1; GCAT; GPD2; HADHA; HCCS; HMGCS2; } \\
\text { IDH3A; KIAA0218; LARS2; LYPLA1; MLLT2; } \\
\text { MRPL39; MTIF2; MTX1; NDUFS7; NDUFV1; } \\
\text { NOX5; NS; NUDT1; NUP155; OGDH; PC; PRDX5; } \\
\text { PRSS25; QP-C; RAF1; SH3GLB1; SLC25A12; } \\
\text { SLC25A21; SLC25A4; SLC37A3; SMYD2; SOD2; } \\
\text { SQRDL; SUPV3L1; TIMM13; TM4SF7; TTC1; } \\
\text { TTC11; TXN2; VAV1; YME1L1 }\end{array}$ \\
\hline Ribosome-associated & 0.008 & $\begin{array}{l}\text { RPL17; RPL22; RPL35A; RPL37; RPL41; RPLP1; } \\
\text { RPS13; RPS23; RPS3; RPS3A; UBA52 }\end{array}$ \\
\hline Endosome transport & 0.014 & EHD1; MCOLN1; RAB9B; SNX17; VPS4B; ZFYVE20 \\
\hline Cysteine-type endopeptidase activity & 0.037 & $\begin{array}{l}\text { CASP3; CTSL; CYLD; D13S106E; FIBL-6; HSP70-4; } \\
\text { MGC3162; MPV17; PIGK; USP10; USP3; USP38; } \\
\text { USP39; USP46 }\end{array}$ \\
\hline
\end{tabular}

${ }^{a}$ The EASE score is the default metric used by EASE to rank categories of genes by over-representation. ${ }^{b}$ Genes in the total group of genes assayed that belong to the specific gene category.

to induce angiogenesis; BIRC1 and BIRC6 known to be apoptosis inhibitor factors; SMURF2 which induces the ubiquitination and degradation of $\mathrm{Smad} 2$, which is an important mediator of the antiproliferative signal triggered by TGF- 31 receptor; PIK3 and EIF4eL3 which play an important role in cell proliferation. Genes that proved more highly expressed in normal colon mucosa than in colon cancers are also of considerable interest: for example, B2M and DEFB1 involved in the immune response, CASP3 and DIABLO known to be important mediators of apoptosis.

Validation studies. Quantitative real-time PCR was used to confirm the reliability of the microarray results. We chose 5 sequences found to be up-regulated in colorectal cancer at microarray analysis (SMURF2, EIF4eL3, PIK3, SLC16A1 and F11R) and 5 sequences whose expression appeared to decrease (MT1G, B2M, CASP3, TIMP3 and DSC3). The results from real-time PCR showed changes in gene expression consistent with microarray data (Fig. 2).

HLA-E was chosen for protein expression validation because alterations of it may be involved in an interesting tumor escape strategy from immune surveillance in colon cancer tissues. Using a commercial monoclonal antibody, we used Western blotting to compare HLA-E protein expression in cancer vs. mucosa samples. Protein production for analyzed tumor samples was consistent with mRNA levels measured by microarray (Fig. 3).

Furthermore, HLA-E expression and tissue distribution was determined in colorectal cancer and normal mucosa samples by immunohistochemical assay. Strong positivity to anti-HLA-E mAb was seen in epithelial cells, while weak staining for anti-HLA-E mAb was observed in sections of normal mucosa used as negative controls (Fig. 4). In some tissue areas an increase was also detected in HLA-E expression intensity involving the plasma membrane of colon lumen epithelial cells.

\section{Discussion}

Colorectal cancer carcinogenesis implies various different genetic programs that we have tried to clarify by gene expression profiling analysis.

For the first time a group of Argentine CRC patients were studied at a genomic level. Using the cDNA microarray, we examined the differences in overall gene expression profiles in the tumor microenvironment of CRC tissues from 21 patients who underwent surgical resection as compared to normal mucosa. Since molecular analysis of cells in their 
Table IV. The most significant $(\mathrm{p}<0.001)$ known genes that show up- or down-regulation in CRC when compared with normal colon.

\begin{tabular}{|c|c|c|c|}
\hline Gene symbol & $\begin{array}{l}\text { Increased in colon cancer } \\
\text { Gene name }\end{array}$ & Chromosome & $\begin{array}{l}\text { Average fold } \\
\text { change }^{\mathrm{a}}\end{array}$ \\
\hline ZNF207 & Zinc finger protein 207 & $17 \mathrm{q} 12$ & 4.28 \\
\hline HLA-E & Major histocompatibility complex, class I, E & $6 \mathrm{p} 21.3$ & 4.26 \\
\hline XPNPEP1 & X-prolyl aminopeptidase (aminopeptidase P) 1, soluble & $10 \mathrm{q} 25.3$ & 4.09 \\
\hline PIK3R2 & Phosphoinositide-3-kinase, regulatory subunit, (p85 ß) & $19 q 13.2-q 13.4$ & 3.91 \\
\hline EIF4EL3 & Eukaryotic translation initiation factor $4 \mathrm{E}$-like 3 & $2 q 37.1$ & 3.57 \\
\hline FXR2 & Fragile X mental retardation, autosomal homolog 2 & $17 \mathrm{p} 13.1$ & 3.45 \\
\hline ATF7 & Activating transcription factor 7 & $12 \mathrm{q} 13$ & 3.41 \\
\hline VTI1B & Vesicle transport through interaction with t-SNAREs homolog $1 \mathrm{~B}$ & $14 \mathrm{q} 24.1$ & 3.32 \\
\hline PICALM & Phosphatidylinositol binding clathrin assembly protein & $11 \mathrm{q} 14$ & 3.14 \\
\hline BIRC1 & Baculoviral IAP repeat-containing 1 & $5 q 13.1$ & 3.13 \\
\hline UBE2B & Ubiquitin-conjugating enzyme E2B (RAD6 homolog) & $5 q 23-q 31$ & 3.11 \\
\hline LATS1 & LATS, large tumor suppressor, homolog 1 (Drosophila) & $6 q 24-q 25.1$ & 3.11 \\
\hline FKBP9 & FK506 binding protein $9,63 \mathrm{kDa}$ & $7 \mathrm{p} 11.1$ & 3.00 \\
\hline LAP1B & Lamina-associated polypeptide 1B & $1 \mathrm{q} 24.2$ & 2.95 \\
\hline RAB3-GAP150 & rab3 GTPase-activating protein, non-catalytic subunit (150 kDa) & $1 \mathrm{q} 42.11$ & 2.95 \\
\hline HDLBP & High density lipoprotein binding protein (vigilin) & $2 q 37$ & 2.89 \\
\hline SLC7A2 & Solute carrier family 7 (cationic amino acid transporter, y+ system) & $8 \mathrm{p} 22-\mathrm{p} 21.3$ & 2.88 \\
\hline PTPN12 & Protein tyrosine phosphatase, non-receptor type 12 & $7 q 11.23$ & 2.85 \\
\hline PIGM & Phosphatidylinositol glycan, class $\mathrm{M}$ & $1 \mathrm{q} 23.1$ & 2.84 \\
\hline KRT7 & Keratin 7 & $12 q 12-q 13$ & 2.83 \\
\hline IVD & Isovaleryl coenzyme A dehydrogenase & $15 q 14-q 15$ & 2.76 \\
\hline DDX3X & DEAD/H (Asp-Glu-Ala-Asp/His) box polypeptide 3 & $\mathrm{Xp} 11.3-\mathrm{p} 11.23$ & 2.75 \\
\hline PTPN12 & Protein tyrosine phosphatase, non-receptor type 12 & $7 \mathrm{q} 11.23$ & 2.69 \\
\hline RNF19 & Ring finger protein 19 & $8 q 22$ & 2.65 \\
\hline SMURF2 & E3 ubiquitin ligase SMURF2 & $17 q 22-q 23$ & 2.63 \\
\hline ENC1 & Ectodermal-neural cortex (with BTB-like domain) & $5 q 12-q 13.3$ & 2.62 \\
\hline SAE1 & SUMO-1 activating enzyme subunit 1 & $19 q 13.33$ & 2.61 \\
\hline DONSON & Downstream neighbor of SON & $21 \mathrm{q} 22.1$ & 2.57 \\
\hline cig5 & Viperin & $2 \mathrm{p} 25.2$ & 2.57 \\
\hline LBP-32 & LBP protein 32 & $2 \mathrm{p} 25.1$ & 2.57 \\
\hline NAPE-PLD & & & 2.56 \\
\hline ZNF275 & Zinc finger protein 275 & $\mathrm{Xq} 28$ & 2.54 \\
\hline UBE2D3 & Ubiquitin-conjugating enzyme E2D 3 (UBC4/5 homolog, yeast) & $4 \mathrm{q} 24$ & 2.53 \\
\hline SMARCE1 & SWI/SNF related, matrix associated & $17 \mathrm{q} 21.2$ & 2.49 \\
\hline SEC22L3 & SEC22 vesicle trafficking protein-like 3 (S. cerevisiae) & $3 \mathrm{p} 21.33$ & 2.46 \\
\hline GTPBP5 & GTP binding protein 5 (putative) & $20 q 13.33$ & 2.43 \\
\hline MLL3 & Myeloid/lymphoid or mixed-lineage leukemia3 & $7 q 34-q 36$ & 2.43 \\
\hline ATM & Ataxia telangiectasia mutated (includes groups A, C and D) & $11 q 22-q 23$ & 2.39 \\
\hline ZNF496 & & & 2.38 \\
\hline DDX51 & Dead box protein 73D-like & $12 q 24.33$ & 2.35 \\
\hline PCYOX1 & & & 2.34 \\
\hline WDR31 & & & 2.34 \\
\hline VEGF & Vascular endothelial growth factor & $6 \mathrm{p} 12$ & 2.33 \\
\hline $\mathrm{ZCCHC} 4$ & HSPC052 protein & $4 \mathrm{p} 15.31$ & 2.30 \\
\hline DISC1 & Disrupted in schizophrenia 1 & $1 q 42.1$ & 2.30 \\
\hline PMS1 & PMS1 postmeiotic segregation increased 1 (S.cerevisiae) & $2 q 31-q 33$ & 2.26 \\
\hline DPP8 & Dipeptidylpeptidase 8 & $15 q 22$ & 2.25 \\
\hline FBLP-1 & Filamin-binding LIM protein-1 & $1 \mathrm{p} 36.13$ & 2.25 \\
\hline CSPG3 & Chondroitin sulfate proteoglycan 3 (neurocan) & $19 \mathrm{p} 12$ & 2.23 \\
\hline SAMSN1 & SAM domain, SH3 domain and nuclear localisation signals, 1 & $21 \mathrm{q} 11$ & 2.22 \\
\hline BMPR2 & Bone morphogenetic protein receptor, type II (serine/threonine kinase) & $2 q 33-q 34$ & 2.20 \\
\hline PAICS & Phosphoribosylaminoimidazole carboxylase & 4pter-q21 & 2.18 \\
\hline SLC16A1 & Solute carrier family 16 (monocarboxylic acid transporters) member 1 & $1 p 12$ & 2.18 \\
\hline ARPC1B & Actin related protein $2 / 3$ complex, subunit $1 \mathrm{~B}, 41 \mathrm{kDa}$ & $7 q 22.1$ & 2.17 \\
\hline
\end{tabular}


Table IV. Continued.

\begin{tabular}{|c|c|c|c|}
\hline Gene symbol & $\begin{array}{l}\text { Increased in colon cancer } \\
\text { Gene name }\end{array}$ & Chromosome & $\begin{array}{l}\text { Average fold } \\
\text { change }^{\mathrm{a}}\end{array}$ \\
\hline HNRPU & Heterogeneous nuclear ribonucleoprotein U (scaffold attachment factor A) & $1 \mathrm{q} 44$ & 2.17 \\
\hline BIRC6 & Baculoviral IAP repeat-containing 6 (apollon) & $2 \mathrm{p} 22-\mathrm{p} 21$ & 2.16 \\
\hline PC326 & PC326 protein & $1 \mathrm{q} 23.3$ & 2.15 \\
\hline PITPNB & Phosphotidylinositol transfer protein, $\beta$ & $22 \mathrm{q} 12.1$ & 2.15 \\
\hline ZNF450 & Zinc finger protein 450 & $6 q 21$ & 2.14 \\
\hline PIPOX & Pipecolic acid oxidase & $17 \mathrm{q} 11.2$ & 2.13 \\
\hline SCA2 & Spinocerebellar ataxia 2 & $12 \mathrm{q} 24$ & 2.13 \\
\hline RB1CC1 & RB1-inducible coiled-coil 1 & $8 \mathrm{p} 22-\mathrm{q} 21.13$ & 2.12 \\
\hline CALM1 & Calmodulin 1 (phosphorylase kinase, delta) & $14 q 24-q 31$ & 2.12 \\
\hline ZNF146 & Zinc finger protein 146 & $19 \mathrm{q} 13.1$ & 2.12 \\
\hline ZNF169 & Zinc finger protein 169 & $9 q 22$ & 2.09 \\
\hline PDCD4 & Programmed cell death 4 (neoplastic transformation inhibitor) & $10 \mathrm{q} 24$ & 2.08 \\
\hline CNTNAP2 & Contactin associated protein-like 2 & $7 q 35-q 36$ & 2.08 \\
\hline DDX54 & DEAD box helicase $97 \mathrm{kDa}$ & $12 \mathrm{q} 24.21$ & 2.07 \\
\hline ITGA2 & Integrin, $\alpha 2$ (CD49B, $\alpha 2$ subunit of VLA-2 receptor) & $5 q 23-q 31$ & 2.06 \\
\hline HIST1H2AC & Histone $1, \mathrm{H} 2 \mathrm{ac}$ & $6 \mathrm{p} 21.3$ & 2.05 \\
\hline PSG9 & Pregnancy specific $\beta$-1-glycoprotein 9 & $19 q 13.2$ & 2.05 \\
\hline SLC35E3 & Solute carrier family 35 , member E3 & $12 q 15$ & 2.04 \\
\hline STK36 & Serine/threonine kinase 36 (fused homolog, Drosophila) & $2 q 35$ & 2.03 \\
\hline PDZGEF1 & PDZ domain containing guanine nucleotide exchange factor (GEF) 1 & $4 q 32.1$ & 2.02 \\
\hline ITGAL & Integrin, $\alpha$ L [antigen CD11A (p180)], lymphocyte function-associated & $16 \mathrm{p} 11.2$ & 2.01 \\
\hline SLC2A11 & Solute carrier family 2 (facilitated glucose transporter), member 11 & & 2.00 \\
\hline Gene symbol & $\begin{array}{l}\text { Decreased in colon cancer } \\
\text { Gene name }\end{array}$ & Chromosome & $\begin{array}{l}\text { Average fold } \\
\text { change }^{\mathrm{a}}\end{array}$ \\
\hline B2M & B-2-microglobulin & $15 q 21-q 22.2$ & -2.82 \\
\hline CA2 & Carbonic anhydrase II & $8 \mathrm{q} 22$ & -2.30 \\
\hline YT521 & Splicing factor YT521-B & $4 q 13.3$ & -2.21 \\
\hline MT1G & Metallothionein 1G & $16 q 13$ & -2.20 \\
\hline NCKAP1 & NCK-associated protein 1 & $2 \mathrm{q} 32$ & -2.12 \\
\hline S100A10 & S100 calcium binding protein A10 (annexin II ligand) & $1 \mathrm{q} 21$ & -2.11 \\
\hline ATE1 & Arginyltransferase 1 & $10 q 26.13$ & -2.10 \\
\hline RRAS2 & Related RAS viral (r-ras) oncogene homolog 2 & $11 \mathrm{p} 15.2$ & -2.07 \\
\hline POLYDOM & Likely ortholog of mouse polydom & $9 \mathrm{q} 31-\mathrm{q} 32$ & -2.05 \\
\hline TFPI & Tissue factor pathway inhibitor (lipoprotein-associated) & $2 q 31-q 32.1$ & -2.04 \\
\hline NF2 & Neurofibromin 2 (bilateral acoustic neuroma) & $22 \mathrm{q} 12.2$ & -2.02 \\
\hline CNNM3 & Cyclin M3 & 2p12-p11.2 & -2.02 \\
\hline
\end{tabular}

${ }^{a}$ The averages of tumor to reference fold changes (mean of 21 values) of fluorescence are reported for the selected genes ( $\left.\mathrm{p}<0.001\right)$. In bold are validated genes by qRT-PCR or immunoblotting.

native tissue environment provides the most accurate picture of the in vivo disease state, we preferred to analyze whole cancer tissues, instead of micro-dissecting malignant cells alone. We believed this would lead to a better understanding of colorectal cancer biology and its interactions with both extracellular matrix and immune system cellular elements. Moreover, since we established an RNA pool of mucosa as a standard reference to compare tumor expression, similar future studies in our laboratory will be available for comparison.
In the present study, comparing the gene expression profiles of colorectal cancer and normal mucosa we were able to identify 1,306 known genes that displayed different levels of expression (defined as $>1.5$-fold up- or down-regulation) and were ascribable to a broad range of functional categories. Of the up-regulated genes, the most represented cluster belonged to inositol/phosphatidylinositol kinase activity (Table II). This finding is especially noteworthy, since genetic alterations of the phosphatidylinositol 3-kinase (PI3-kinase) pathway 

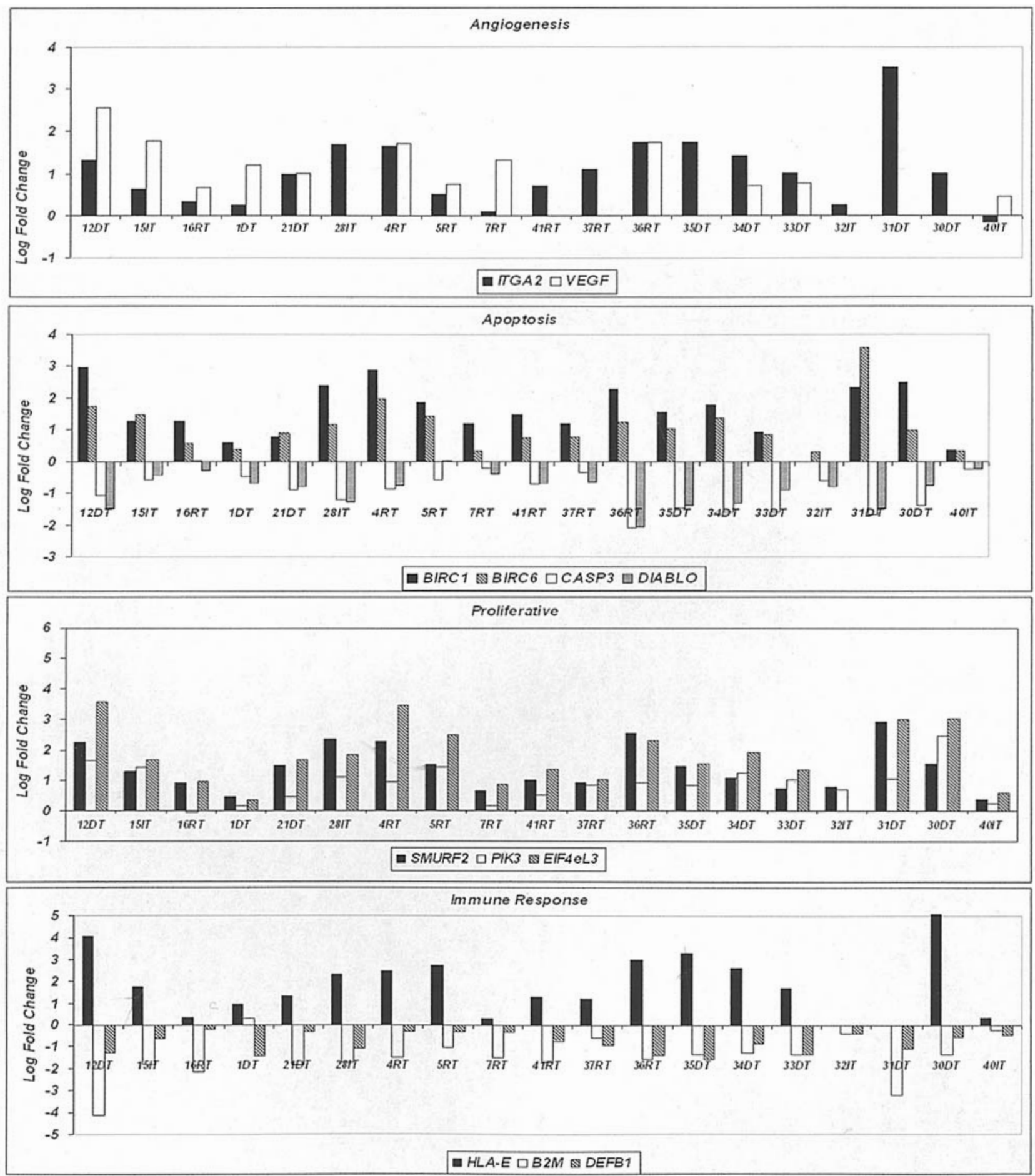

Figure 1. Graphical overview describing functional categories of known genes with increasing or decreasing expression from normal tissue to tumor in at least $80 \%$ of analyzed patients. The majority of genes involved in angiogenesis, cell proliferation and anti-apoptotic signal show increasing expression. Details are available in the supplementary data (see the GEO website at http: //www.ncbi.nlm.nih.gov/projects/geo/; accession number: GSE3294).

have been detected in diverse human cancers (13). PI3Ks, which consist of an $85 \mathrm{kDa}$ regulatory subunit and a catalytic $110 \mathrm{kDa}$ subunit, play critical roles in mitogenic signaling; the alteration of this pathway has been shown to play a pivotal role in the tumorigenesis of colon cancers (14-16). Inhibition of the PI3K signaling pathway components by chemical agents could be a target area for drug development in the treatment of colon cancer. The second most represented gene category among up-regulated genes was apoptosis inhibitor activity. Deregulation of apoptotic pathways contributes to neoplastic diseases by preventing or delaying normal cell turnover, thus promoting cell accumulation. Defects in apoptosis also facilitate tumor progression, by rendering cancer cells resistant to death mechanisms relevant to metastasis (17). In our study, a cluster of 5 anti-apoptotic genes (Table II) were up-regulated in cancer cells; of these, BIRC1 and BIRC6 encode for proteins known as IAPs (inhibitor of apoptosis proteins) which represent a family of 


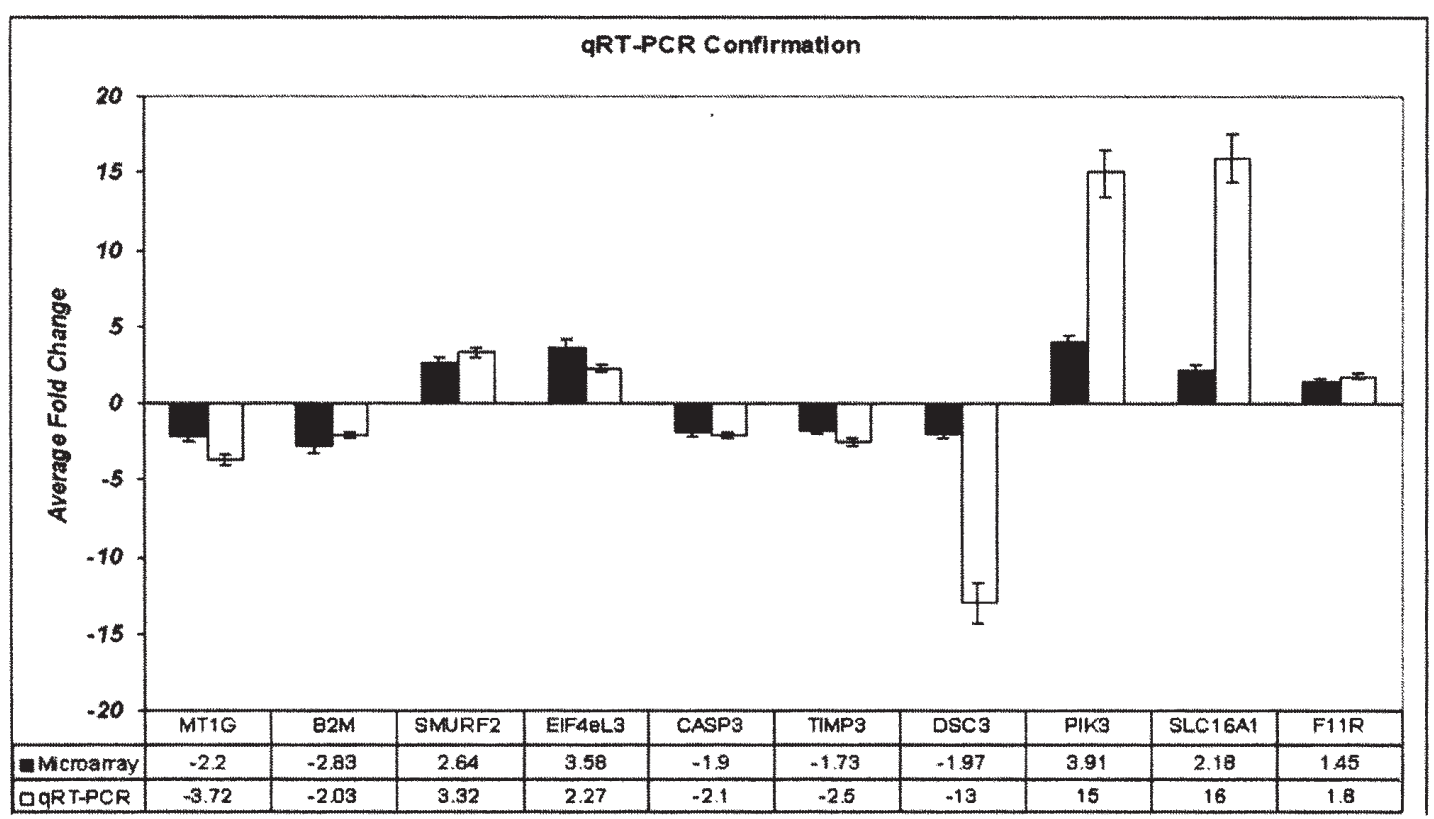

Figure 2. Comparison of microarray analyses with quantitative real-time PCR analyses using gene specific primers (Table I). Expression analyses are shown for 10 selected genes using pools of normal tissue and single samples of Dukes' stage B and C adenocarcinomas (6 patients were analyzed). The y-axis shows expression intensity 'fold changes' derived from microarray and qRT-PCR respectively. The error bars indicate the variations between the single determinations.

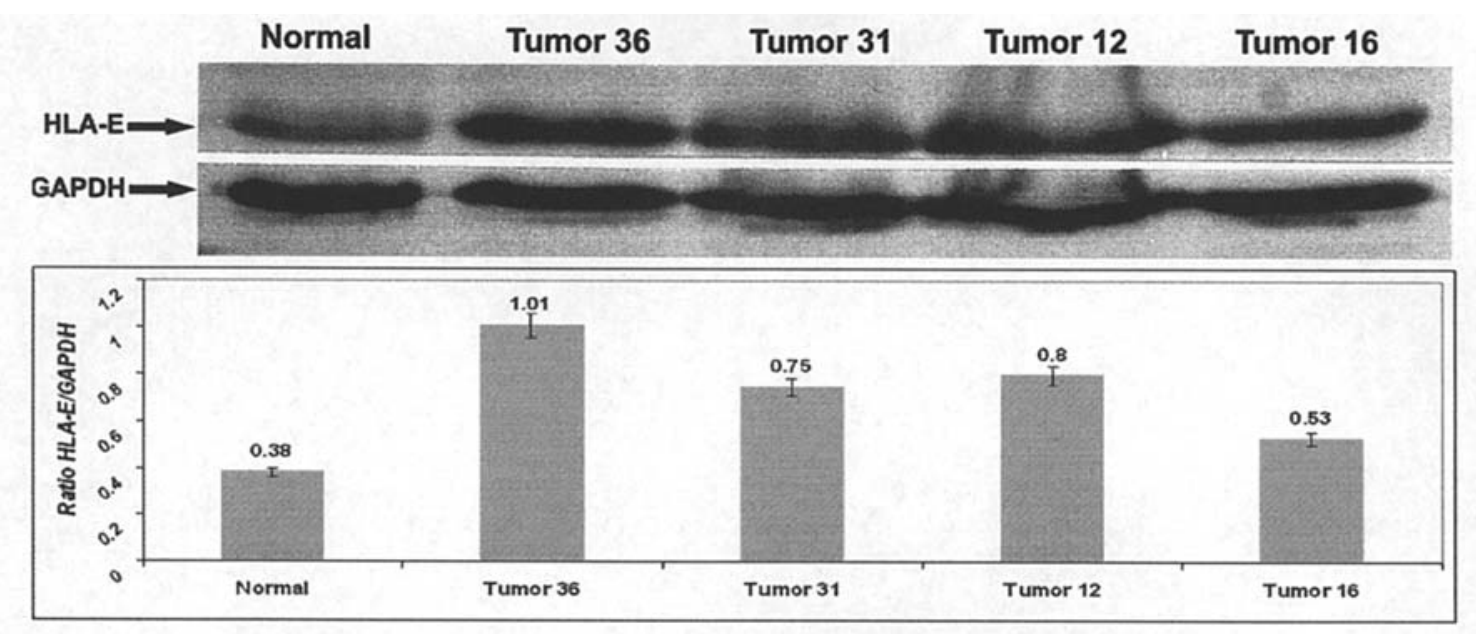

Figure 3. Western blot analysis of whole lysates of human colon cancer tissues (sample numbers of tumors refer to samples previously analyzed on microarrays) and normal colon mucosa (pool of 6 non-cancerous tissues) with the aim of assessing the relative change in HLA-E concentration. Immunodetection was carried out as described in Materials and methods. As shown in the figure, HLA-E ( 43 kDa) expression was increased (corrected for GAPDH expression) in all analyzed tumor tissues. Relative band intensity quantification was carried out by Image J v1.34n (NIH, USA).

evolutionarily conserved apoptosis suppressors (18). IAPs are known to act as endogenous inhibitors of the cell death proteases, directly suppressing caspases. This mechanism could represent a tumor strategy for achieving apoptosis resistance (19). Consistent with this repressed apoptotic phenotype we found that an endogenous antagonist of the IAPs, named DIABLO, was down-regulated in our colon cancer samples. DIABLO is known to keep the apoptosissuppressor inhibited thus resulting in a more repressed apoptotic phenotype $(20,21)$. From such results it appears evident that up-regulated genes in CRC are mainly involved in two major physiological pathways, i.e. cell proliferation and apoptosis. Emerging knowledge on the proteins that promote proliferative and anti-death signals may afford multiple new opportunities for therapy, including discovery and development of new anticancer agents, as well as potentially more effective exploitation of existing ones.

The genes that we identified as being under-expressed in colon tumor versus normal mucosa are involved in a variety of functions but essentially encode for proteins that may be grouped under two main functional headings: the mitochondrial protein family and the ribosome family (Table II). It has been shown previously that the mitochondrion, long considered an organelle specific to energy metabolism, is in fact multi-functional and involved in many diseases $(22,23)$. As already hypothesized by Ohta (24) mitochondrial DNA 

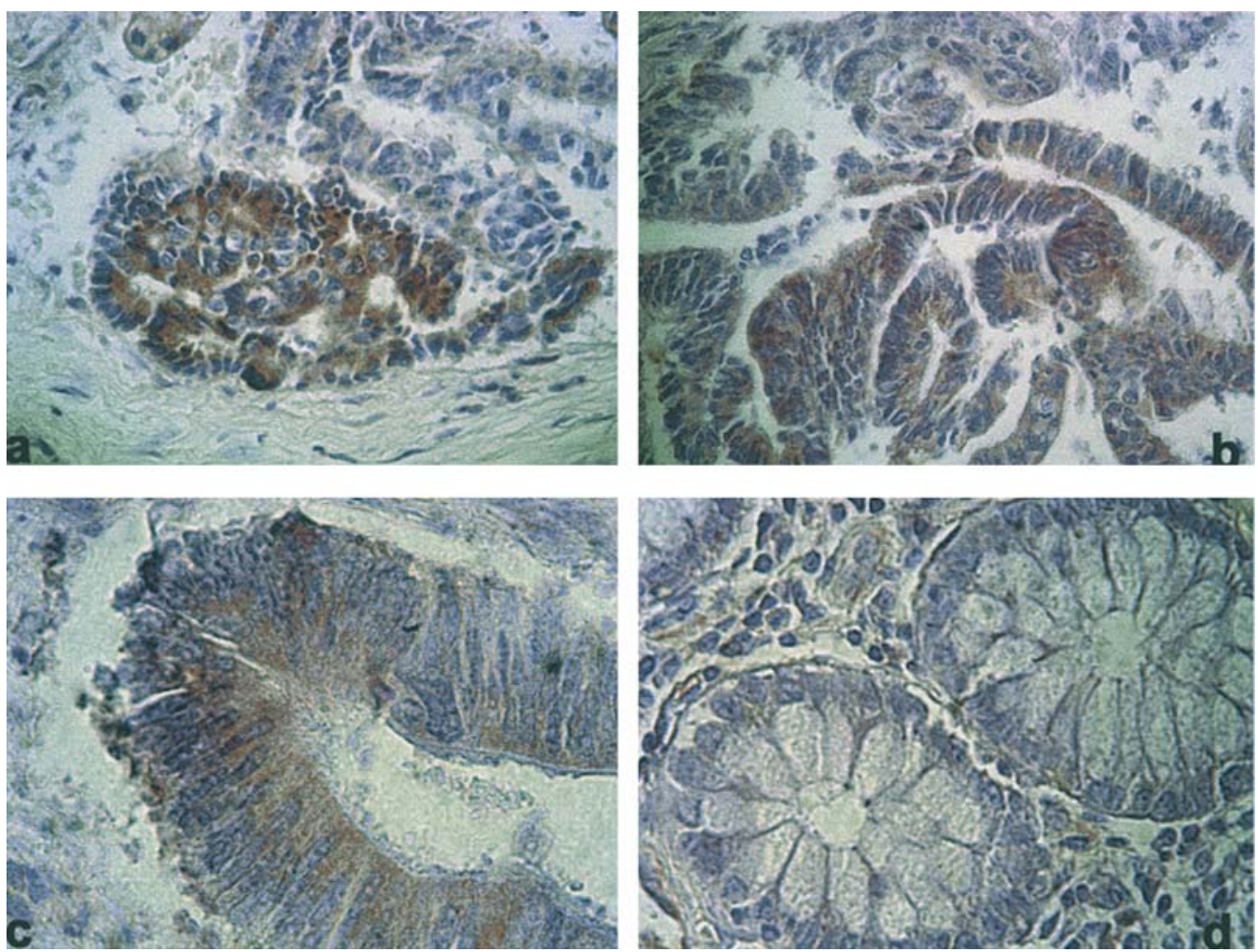

Figure 4. Immunohistochemistry of formalin-fixed paraffin-embedded sections of Dukes' B and C adenocarcinomas and their matching normal mucosa. MEM-E/02 (BioVendor Laboratory Medicine, Inc.) mAb for HLA-E molecule was first tested at several dilutions in order to determine the optimal dilution to be used. Non-specific binding was prevented by applying 10\% normal horse serum in PBS for 20 min at room temperature. Slide sections were incubated with primary Ab diluted 1:50 overnight at $4^{\circ} \mathrm{C}$. With human anti-HLA-E antibody there is strong staining in the luminal epithelium of cancer tissues (a-c) but no significant staining in normal colon sections (d). As shown at a higher magnification (c), epithelial cells in the colon sections are positively stained with enhanced staining of the membrane surface.

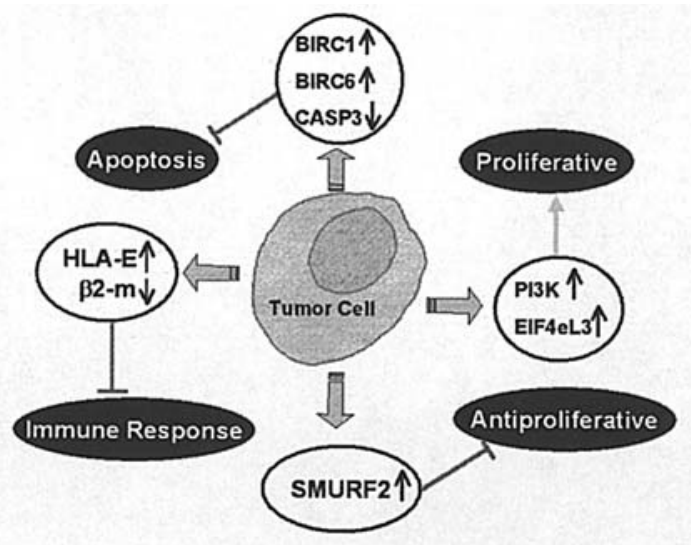

Figure 5. Overview of cancer gene pathways. The major pathways regulating cell birth, cell death and immune response are depicted as black ovals. The schema emphasizes the genes that have been shown to be altered in CRC human tumors, though many other genes participate in these pathways. Additionally, some of these genes appear in more than one pathway and there is substantial 'cross-talk' between pathways. More detailed information on these pathways can be found in several comprehensive reviews (41).

accumulates somatic mutations during the progression of cancer. Although little is known about the contribution of mitochondrial mutations to carcinogenesis, one possible cause of CRC carcinogenesis, as suggested by BirkenkampDemtroder et al (25) might be located at a mitochondrion level, where DNA damage accumulates more rapidly.
Although increased protein synthesis is usually associated with neoplastic transformation, we found that many genes encoding for ribosomal proteins were decreased in cancer tissue. Since ribosome biogenesis and translation control are essential cellular processes governed at numerous different levels $(26,27)$, it could be speculated that the expression pattern for genes involved in these processes is tumorspecific. It may plausibly be imagined that alterations to the protein synthesis machinery underlie the cancer phenotype. Consistent with this hypothesis, we found several genes involved in translational control, such as eukaryotic translation initiation factor 4e-like 3 (eIF4eL3), which shows a significant over-expression in cancer tissue with the highest level of up-regulation in stage III colorectal adenocarcinomas. eIF-4eL3 is a rate-limiting factor for the initiation of cap-dependent mRNA translation by the eIF-4F translation initiation complex, which in turn dramatically impacts upon the quantitative expression of key malignancy-related genes $(28,29)$. elF-4eL3 acts like elF-4e, which is known to be overexpressed in various solid tumors, including breast, bladder, cervical, and head and neck cancers (30) and only barely detectable in certain highly proliferative and aggressive neoplasms (e.g. squamous cell carcinoma of the lung and melanoma) (31). It is hence reasonable to consider elF-4eL3 itself in the same light. This, and other data from the literature suggesting an eIF-4e role in colon tumorigenesis (32) make it a possible new marker for use in chemotherapy intervention studies in patients with colorectal cancer. 
One intriguing aspect, usually unnoticed when considering specific carcinogenesis, is the association between oncogenic transformation and alteration of various components of the immunological machinery. In our study, the top-ranking genes across all samples studied were the two elements, known to be involved in immune system machinery: human leukocyte antigen E (HLA-E) and B2-microglobulin (B2M). As can be seen from Table IV, B2M was the most decreased gene while HLA-E was the second most increased gene in a CRC environment. No previous work was found in the literature describing this simultaneous HLA-E up-regulation and B2M down-regulation. Such an expression pattern might hypothetically represent a tumor escape strategy in colorectal cancer. In this connection, HLA-class I molecules are composed of two non-covalently associated glycoproteins. The larger 44-kDa heavy chain is the product of HLA-A, -B, and -C (class Ia) and HLA-E, -F, and -G (class Ib) genes, while the smaller $12-\mathrm{kDa}$ chain is the product of the 32 -microglobulin gene. A complex of these two glycoproteins together with a peptide formed from degraded endogenous proteins, is expressed at the cell surface to enable the peptide to be presented to specific receptors on the surface of cytotoxic $\mathrm{T}$ cells (33). HLA-class Ib molecules (HLA-E, -F, -G) differ from the classical Ia molecules (HLA-A, -B and -C) in that the former show limited polymorphism, low cell expression and limited tissue distribution. Several studies have shown that some tumors lack cell surface expression of HLA-class Ia molecules (haplotype HLA loss) suggesting that this is one mechanism by which tumor cells escape immune recognition by cytotoxic T cells (34). However, loss of HLA-class Ia surface expression correlates with a better prognosis in colorectal cancer patients - a better prognosis which may be caused by elimination of HLA-negative cells by natural killer (NK) cells (35). Attack by natural killer cells is a balance between positive and negative signals, with different NK cell receptors mediating inhibition or activation respectively $(36,37)$. In this context, as previously demonstrated by others, HLA-E migrates to the cell surface, where it can interact with CD94/NKG2A receptors on natural killer cells $(38,39)$. This interaction inhibits natural killer cell-mediated lysis; in our study we found an increased HLA-E expression in cancer tissue, which is consistent with a less vulnerable tissue to lysis by NK cells. Moreover, we observed simultaneous B2M down-regulation. This finding is quite controversial, since B2M availability gives rise to HLA-E cell surface expression in tumor cell lines (40). However, we hypothesize that the weak expression of $\mathrm{B} 2 \mathrm{M}$ molecule in tumor cells may convert it into a limiting factor for HLA molecule cell surface expression. This event could favor the immune escape mechanism in colon tumor cells of the kind we are studying. In fact, the competition for B2M becomes a critical event, conferring a considerable advantage on HLA-E molecules due to its substantial over-expression in tumor cells. In short, tumor cells exhibit complex HLA-class I alterations that may simultaneously inhibit CTLs, by losing the restriction element, and NK cell activity, by expressing HLA-E at the cell surface.

In conclusion, understanding the molecular alterations in colorectal cancer is urgently needed to define new biomarkers and treatment targets. To the best of our knowledge, this is the first work on over-expression for transcripts such as SMURF2, BIRC1, BIRC6 and eIF-4eL3; no information as to their involvement in $\mathrm{CRC}$ carcinogenesis is available in the literature. According to the data provided here, they might form novel potential tumor targets for chemotherapy strategies (Fig. 5).

Again, interesting insights consistent with a potential tumor escape mechanism from both 'innate' and cellular specific immune response have been reported. Thus far there are no expression studies in vivo on human CRC tissues reporting similar results; the complexity of the immune network and the still enigmatic host-tumor interactions make this task both challenging and fascinating. In particular, the acknowledged synergism between innate and adaptive immune responses is leading to a more comprehensive immunotherapeutic approach, taking into consideration the multiple variables determining the ultimate outcome of the immune response against malignant cells. A real functional meaning for the mechanism caused by HLA-E needs to be validated. Its actual in vivo over-expression should be checked and correlated with the effectiveness of immunotherapy protocols used in CRC patients.

\section{Acknowledgements}

This work was supported with funds from Fundacion Sales, Fundacion Mosoteguy, Fundacion Cancer (FUCA), Agencia Nacional de Promocion Cientifica y Tecnologica, Fondazione CARISBO 'Center for Research into Molecular Genetics' and the University of Buenos Aires. J.M. is a member of CONICET, M.B. is Fellow of the Fundación Sales. We thank Dr Marcela Barrio for her excellent contribution to the discussion of the paper, Mariana Rodriguez Zubieta for her assistance with immunochemistry analysis, Dr A. Tomas and Dr P. Gonzalez (Hospital M.B. de Martinez), Dr Martha Endelman and Dr Porqueres (Hospital Julio Mendez) for their collaboration with CRC collection.

\section{References}

1. Jemal A, Murray T, Samuels A, et al: Cancer statistics. CA Cancer J Clin 53: 5-26, 2003.

2. Pasetto LM, Jirillo A, Iadicicco G, et al: FOLFOX versus FOLFIRI: a comparison of regimens in the treatment of colorectal cancer metastases. Anticancer Res 25: 563-576, 2005.

3. Goldberg RM and Gill S: Recent phase III trials of fluorouracil, irinotecan, and oxaliplatin as chemotherapy for metastatic colorectal cancer. Cancer Chemother Pharmacol 54 (Suppl 1): P57, 2004.

4. Loeve F, van Ballegooijen M, Snel P, et al: Colorectal cancer risk after colonoscopic polypectomy: a population-based study and literature search. Eur J Cancer 41: 416-422, 2005.

5. Koehler A, Bataille F, Schmid C, et al: Gene expression profiling of colorectal cancer and metastases divides tumours according to their clinicopathological stage. J Pathol 204: 65-74, 2004.

6. Kolonel LN, Altshuler D and Henderson BE: The multiethnic cohort study: exploring genes, lifestyle and cancer risk. Nat Rev Cancer 4: 519-527, 2004.

7. Conlin A, Smith G, Carey FA, et al: The prognostic significance of K-ras, p53 and APC mutations in colorectal carcinoma. Gut 54: 1283-1286, 2005.

8. Croner RS, Foertsch T, Brueckl WM, et al: Common denominator genes that distinguish colorectal carcinoma from normal mucosa. Int J Colorectal Dis 20: 353-362, 2005.

9. Raetz EA and Moos PJ: Impact of microarray technology in clinical oncology. Cancer Invest 22: 312-320, 2004. 
10. Chiu ST, Hsieh FJ, Chen SW, et al: Clinicopathologic correlation of up-regulated genes identified using cDNA microarray and real-time reverse transcription-PCR in human colorectal cancer. Cancer Epidemiol Biomarkers Prev 14: 437-443, 2005.

11. Nambiar PR, Nakanishi M, Gupta R, et al: Genetic signatures of high- and low-risk aberrant crypt foci in a mouse model of sporadic colon cancer. Cancer Res 64: 6394-6401, 2004.

12. Mariadason JM, Arango D, Shi Q, et al: Gene expression profiling-based prediction of response of colon carcinoma cells to 5-fluorouracil and camptothecin. Cancer Res 63: 8791-8812, 2003.

13. Osaki M, Oshimura M and Ito H: PI3K-Akt pathway: its functions and alterations in human cancer. Apoptosis 9: 667-676, 2004

14. Cantley LC: The phosphoinositide 3-kinase pathway. Science 296: 1655-1657, 2002.

15. Vivanco I and Sawyers CL: The phosphatidylinositol 3-kinase AKT pathway in human cancer. Nat Rev Cancer 2: 489-501, 2002.

16. Sheng H, Shao J, Townsend CM Jr, et al: Phosphatidylinositol 3-kinase mediates proliferative signals in intestinal epithelial cells. Gut 52: 1472-1478, 2003.

17. Watson AJ: Apoptosis and colorectal cancer. Gut 53: 1701-1709, 2004.

18. La Casse EC, Baird S, Korneluk RG, et al: The inhibitors of apoptosis (IAPs) and their emerging role in cancer. Oncogene 17: 3247-3259, 1998.

19. Huang Q, Deveraux QL, Maeda S, et al: Evolutionary conservation of apoptosis mechanisms: lepidopteran and baculoviral inhibitor of apoptosis proteins are inhibitors of mammalian caspase-9. Proc Natl Acad Sci USA 97: 1427-1432, 2000

20. Verhagen AM and Vaux DL: Cell death regulation by the mammalian IAP antagonist Diablo/Smac. Apoptosis 7: 163-166, 2002.

21. Grossmann J, Walther K, Artinger M, et al: Induction of apoptosis before shedding of human intestinal epithelial cells. Am J Gastroenterol 97: 1421-1428, 2002.

22. Green DR and Kroemer G: The pathophysiology of mitochondrial cell death. Science 305: 626-629, 2004.

23. Cuezva JM, Krajewska M, De Heredia ML, et al: The bioenergetic signature of cancer: a marker of tumor progression. Cancer Res 62: 6674-6681, 2002.

24. Ohta S: A multi-functional organelle mitochondrion is involved in cell death, proliferation and disease. Curr Med Chem 10: 2485-2494, 2003.

25. Birkenkamp-Demtroder K, Lotte Christensen L, Harder Olesen S, et al: Gene expression in colorectal cancer. Cancer Res 62: 4352-4363, 2002.

26. Clemens MJ: Targets and mechanisms for the regulation of translation in malignant transformation. Oncogene 23: 3180-3188, 2004.
27. Bertucci F, Salas S, Eysteries S, et al: Gene expression profiling of colon cancer by DNA microarrays and correlation with histoclinical parameters. Oncogene 23: 1377-1391, 2004.

28. Zimmer SG, DeBenedetti A and Graff JR: Translational control of malignancy: the mRNA cap-binding protein, eIF-4E, as a central regulator of tumor formation, growth, invasion and metastasis. Anticancer Res 20: 1343-1351, 2000.

29. Joshi B, Cameron A and Jagus R: Characterization of mammalian eIF4E-family members. Eur J Biochem 271: 2189-2203, 2004.

30. Sorrells DL, Meschonat C, Black D, et al: Pattern of amplification and overexpression of the eukaryotic initiation factor 4E gene in solid tumor. J Surg Res 85: 37-42, 1999.

31. DeBenedetti A and Graff JR: eIF-4E expression and its role in malignancies and metastases. Oncogene 23: 3189-3199, 2004.

32. Berkel HJ, Turbat-Herrera EA, Shi R, et al: Expression of the translation initiation factor eIF4E in the polyp-cancer sequence in the colon. Cancer Epidemiol Biomarkers Prev 10: 663-666, 2001.

33. Townsend A, Ohlen C, Bastin J, et al: Association of class I major histocompatibility heavy and light chains induced by viral peptides. Nature 340: 443-448, 1989.

34. Smith ME, Bodmer WF and Bodmer JG: Selective loss of HLA$\mathrm{A}, \mathrm{B}, \mathrm{C}$ locus products in colorectal adenocarcinoma. Lancet $\mathrm{i}$ : 823-824, 1988.

35. Kuppen PJ, Gorter A, Hagenaars M, et al: Role of NK cells in adoptive immunotherapy of metastatic colorectal cancer in a syngeneic rat model. Immunol Rev 184: 236-243, 2001.

36. Sandel MH, Speetjens FM, Menon AG, et al: Natural killer cells infiltrating colorectal cancer and MHC class I expression. Mol Immunol 42: 541-546, 2005.

37. Watson NF, Ramage JM, Madjd Z, et al: Immunosurveillance is active in colorectal cancer as downregulation but not complete loss of MHC class I expression correlates with a poor prognosis. Int J Cancer 118: 6-10, 2005.

38. O'Callaghan CA: Molecular basis of human natural killer cell recognition of HLA-E (human leucocyte antigen-E) and its relevance to clearance of pathogen-infected and tumour cells. Clin Sci 99: 9-17, 2000.

39. Algarra I, Garcia-Lora A, Cabrera T, et al: The selection of tumor variants with altered expression of classical and non-classical MHC class I molecules: implications for tumor immune escape. Cancer Immunol Immunother 53: 904-910, 2004.

40. Marín R, Ruiz-Cabello F, Pedrinaci S, et al: Analysis of HLA-E expression in human tumors. Immunogenetics 54: 767-775, 2003.

41. Vogelstein B and Kinzler KW: Cancer genes and the pathways they control. Nat Med 10: 789-799, 2004. 